\title{
Tendência da incidência de câncer do colo do útero invasor em quatro capitais brasileiras: dados dos registros de câncer de base populacional, 1990-2004
}

\section{Trends in incidence of cancer of the cervix intruder in four Brazilian cities: data from population-based cancer registries, 1990-2004}

\author{
Andréia Rodrigues Gonçalves Ayres ${ }^{1}$, Gulnar Azevedo e Silva², Raphael Mendonça Guimarães ${ }^{3}$
}

\begin{abstract}
Resumo
Os Registros de Câncer de Base Populacional (RCBP) coletam sistematicamente dados de serviços de diagnóstico e tratamento de pacientes por câncer, fornecendo assim dados de incidência de câncer. No Brasil, os primeiros RCBP surgiram em Recife e São Paulo na década de 1960, e atualmente existem 28 distribuídos entre capitais e demais cidades pelo país. O objetivo do estudo é Analisar a tendência das taxas de incidência do câncer do colo do útero com base nos dados de RCBP selecionados. Foram acessados os dados disponibilizados no site do Instituto Nacional de Câncer (INCA) dos RCBP de Fortaleza, Porto Alegre, Recife e São Paulo, para obtenção do número de casos para neoplasia maligna do colo do útero, neoplasia do útero porção não especificada e carcinoma in situ do colo do útero por idade. Foram utilizados os denominadores populacionais informados pelo Ministério da Saúde (Datasus). Foram calculadas as taxas específicas por idade para os grupos etários estratificados de 25 a 34, 35 a 49 e 50 a 59 anos. A tendência foi analisada por regressão polinomial. Os períodos com informações disponíveis de 1990 a 2004 variaram de 7 a 13 anos. A análise gráfica mostrou menor regularidade para Recife. A tendência de incidência para o câncer do colo do útero foi declinante e pode ser, parcialmente, explicada em função do tempo entre mulheres de 35 a 59 anos em Fortaleza (CID-10 C53: 35 a 49 anos $\beta=-9.11, p<0,001$ e 50 a 59 anos $\beta=-4.53, p=0.02$ ) e São Paulo (CID-10 C53: 35 a 49 anos $\beta=-4.83, p=0.04$ e 50 a 59 anos $\beta=-4.49, p<0.001$ ). A tendência de incidência de câncer de útero - porção não especificada - mostrou decréscimo entre mulheres de 35 a 59 anos na cidade de Porto Alegre (35-49 anos: $p=0.032 ; 35$ a 49 anos: $p=0.004$ ). A análise da tendência do carcinoma in situ do colo do útero foi crescente em todas as cidades e grupos etários estudados, com exceção de Recife. A regularidade e a cobertura dos RCBP podem interferir na qualidade das estimativas de incidência, porém mesmo com essas limitações, as informações geradas por eles podem ser relevantes para o monitoramento do câncer. A queda observada do câncer invasor e o crescimento do carcinoma in situ em Porto Alegre e Fortaleza podem indicar resultados positivos das ações de rastreamento para o câncer do colo do útero nessas cidades.
\end{abstract}

Palavras-chave: neoplasias do colo do útero; séries temporais; epidemiologia; incidência.

\begin{abstract}
Cancer Registries Population-Based (RCBP) systematically collect data on diagnostic services and treatment of cancer patients thus providing data on cancer incidence. In Brazil, the first PBCR appeared in Recife and São Paulo in the 1960s, and currently there are 28 distributed between capital and other cities across the country. The aim of the study was to analyze
\end{abstract}

Trabalho realizado no Instituto de Medicina Social/Universidade do Estado do Rio de Janeiro (UERJ) - Rio de Janeiro (RJ), Brasil. ${ }^{1}$ Mestre em Saúde Coletiva; Enfermeira do Hospital Universitário Gaffrée e Guinle - Rio de Janeiro (RJ), Brasil.

${ }^{2}$ Doutora em Saúde Pública; Professora Associada do Instituto de Medicina Social (IMS) da UERJ - Rio de Janeiro (RJ), Brasil.

${ }^{3}$ Doutor em Saúde Coletiva; Professor Adjunto do Instituto de Estudos em Saúde Coletiva (IESC) da Universidade Federal do Rio de Janeiro (UFRJ) Rio de Janeiro (RJ), Brasil.

Endereço para correspondência: Raphael Mendonça Guimarães - Avenida Horácio Macedo, s/n - Cidade Universitária - CEP: 21941-598 - Rio de Janeiro (RJ), Brasil - E-mail: raphael@iesc.ufrj.br

Fonte de financiamento: nenhuma.

Conflito de interesses: nada a declarar. 
trends in incidence rates of cancer of the cervix based on the data selected PBCR. We accessed the data available at the site of the National Cancer Institute (NCl) of the PBCR of Fortaleza, Porto Alegre, Recife and Sao Paulo to obtain the number of cases for malignant neoplasm of the cervix, cancer of the uterus and unspecified part carcinoma in situ of the cervix by age. We used population denominators informed by the Ministry of Health (Datasus). We calculated age-specific rates for age groups stratified were 25-34, 35-49 and 50-59 years. The trend was analyzed by polynomial regression. Periods with information available from 1990 to 2004 ranged from 7 to 13 years. The graphical analysis showed less regularity to Recife. The trend of incidence for cancer of the cervix has been declining and may be partly explained as a function of time among women 35 to 59 years in Fortaleza (ICD-10 C53: 35 to 49 years $\beta=-9.11, p<0.001$ and 50 to 59 years $\beta=-4.53, p=0.02$ ) and São Paulo (ICD-10 C53: 35 to 49 years $\beta=-4.83, p=0.04$ and 50 to 59 years $\beta=-4.49, p<0.001$ ). The trend of incidence of cervical cancer - unspecified part - showed decrease among women 35 to 59 years in Porto Alegre (35 to 49 years: $p=0.032 ; 35-49$ years: $B=-1.74, p=0.004)$. The trend analysis of carcinoma in situ of the cervix has been increasing in all cities and age groups studied, with the exception of Recife. The frequency and coverage of the PBCR can interfere with the quality of the estimates of incidence, but even with these limitations, the information generated by them may be relevant to the monitoring of cancer. The observed decrease of invasive cancer and carcinoma in situ growth in Porto Alegre and Fortaleza may indicate positive results of the actions of screening for cancer of the cervix in these cities.

Keywords: uterine cervical neoplasms; time series studies; epidemiology; incidence.

\section{INTRODUÇÃO}

Os Registros de Câncer de Base Populacional (RCBP) reúnem dados de unidades e sistemas notificadores de serviços de diagnóstico e tratamento de pacientes com câncer no Brasil (hospitais, laboratórios, serviços de diagnóstico e tratamento, públicos e privados), fornecendo subsídios para estimar a incidência dos vários tipos da doença ${ }^{1}$. No país, os primeiros RCBP surgiram em Recife e São Paulo na década de $1960^{2,3}$; atualmente, existem 30 distribuídos entre capitais e demais cidades pelo país, sendo que 20 destes apresentam dados consolidados para o período de pelo menos 1 ano, e para 19 os dados estão disponíveis no site do Instituto Nacional de Câncer ${ }^{4}$. Em muitos países, os registros de câncer, sejam hospitalares ou de base populacional, têm sido utilizados para analisar a situação do câncer nas cidades onde se situam ou mesmo oferecem informações para estudos colaborativos de caráter internacional ${ }^{5,6}$. Em geral, os registros de câncer são de notificação passiva, e para sua maior completude e aproveitamento, precisam de integração com outros sistemas de registros de morbidade de mortalidade, aumentando sua potencial participação na elaboração do perfil epidemiológico do câncer no Brasil ${ }^{7}$.

Entre os tipos de câncer para o qual há informações disponíveis nos RCBP, destaca-se o câncer do colo do útero, ocupando o quinto e sexto lugares em incidência e mortalidade, respectivamente, na população mundial. Entre as mulheres, é o segundo mais incidente e o terceiro mais letal ${ }^{8}$. Estimativas oficiais de incidência de câncer no Brasil apontam que, excluídos os tumores de pele não melanoma, o câncer do colo do útero seria o sexto mais comum e o segundo entre mulheres ${ }^{9,10}$. Ainda que varie muito entre as mulheres das diferentes regiões brasileiras, a prevalência de infecção pelo HPV (papilomavírus humano), causa necessária para o desenvolvimento do câncer do colo do útero ${ }^{11,12}$, é alta mesmo entre as mulheres com citologia normal, desde cerca de 10,0\%, podendo chegar a $24,5 \%$, conforme revisão dos estudos brasileiros sobre prevalência ${ }^{13}$. Outra recente publicação, com análise de um período de quase 30 anos que efetuou correção da magnitude com redistribuição proporcional dos óbitos classificados com "útero - porção não especificada" mostrou que, embora tenha sido o segundo mais frequente entre os óbitos até 2005 no país, a tendência é decrescente em todas as suas regiões ${ }^{14}$. Comparando-se os dados de capitais e demais municípios, apenas para os municípios fora das capitais da Região Nordeste verifica-se aumento das taxas nesse período.

Apesar da ampla disponibilidade dos dados dos RCBP, estes ainda são pouco utilizados para fins científicos e de ensino e pesquisa, e são escassos os trabalhos que reúnem informações sobre incidência de câncer com base nos RCBP ${ }^{15,16}$. O presente estudo tem como objetivo analisar a tendência das taxas de incidência do câncer do colo do útero, com base nos dados de RCBP selecionados em quatro capitais brasileiras, no período de 1990 a 2004.

\section{MÉTODOS}

Foram selecionados para o estudo os Registros de Câncer de Base Populacional de Fortaleza, Porto Alegre, Recife e São Paulo, por serem os com maiores períodos de continuidade de informações ${ }^{4}$. Foram incluídos na análise os casos incidentes de neoplasia maligna do colo do útero (invasor) e neoplasia do útero porção não especificada e, ainda, carcinoma in situ do colo do útero, correspondendo respectivamente aos códigos C53, C55 e D06 da $10^{\mathrm{a}}$ edição da Classificação Estatística Internacional de Doenças e Problemas Relacionados com a Saúde ${ }^{17}$. Foram utilizados os denominadores populacionais informados pelo Ministério da Saúde ${ }^{18}$ e calculadas taxas de incidência segundo grupos etários de 25 a 34, 35 a 49 e 50 a 59 anos.

Para a análise de tendência, foram feitos, inicialmente, os diagramas de dispersão das taxas de incidência padronizadas por idade ao longo do período. A seguir, foi realizado o processo de modelagem, considerando as taxas médias de 
incidência para câncer do colo do útero segundo as CID especificadas como variável dependente (y) e os anos do período de estudo como variável independente (x), utilizando modelos de regressão. Para se evitar a colinearidade entre os termos da equação de regressão, a variável ano de óbito foi transformada em ano-centralizado (ano do óbito subtraindo-se o ano do ponto médio da série histórica) ${ }^{18}$.

O primeiro modelo testado foi o de regressão linear simples $\left(\mathrm{Y}=\mathrm{b}_{0}+\mathrm{b}_{1} \mathrm{X}\right)$ e, posteriormente, foram testados os modelos de ordem maior: segundo grau $\left(\mathrm{Y}=\mathrm{b}_{0}+\mathrm{b}_{1} \mathrm{X}+\mathrm{b}_{2} \mathrm{X}^{2}\right)$, terceiro grau $\left(\mathrm{Y}=\mathrm{b}_{0}+\mathrm{b}_{1} \mathrm{X}+\mathrm{b}_{2} \mathrm{X}^{2}+\mathrm{b}_{3} \mathrm{X}^{3}\right)$, logarítmico $\left(\mathrm{Y}=\log _{\mathrm{b}} \mathrm{X}+\mathrm{b}_{1}{ }^{\mathrm{X}}\right)$ e exponencial $\left(\mathrm{Y}=\mathrm{e}_{0}^{\mathrm{b}}+\mathrm{b}_{1}^{\mathrm{X}}\right)^{18}$.

Comocritériosparaa escolha do melhormodelo, foramconsideradosoníveldesignificânciaeaanálisedosresíduos.Quando mais de um modelo satisfazia os critérios de seleção, optou-se pelo mais simples, definido como sendo o de menor grau. Considerou-se tendência significativa quando o valor $\mathrm{p}<0,05$.

\section{RESULTADOS}

Os RCBP para as capitais incluídas no estudo dispunham de informações com períodos compreendidos entre 1990 e

FORTALEZA

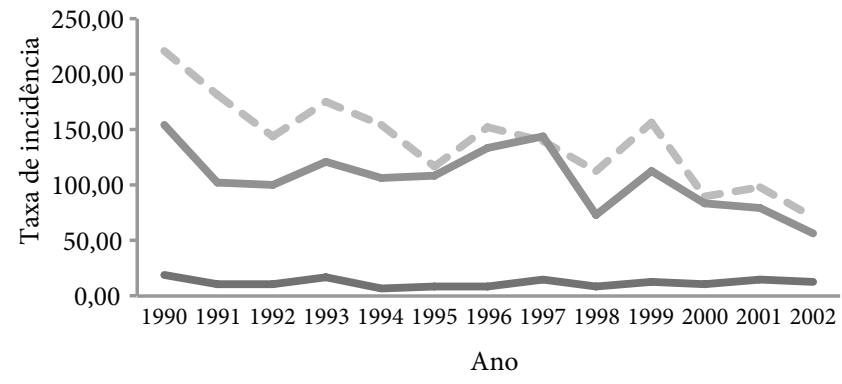

-25 a 34 anos -35 a 49 anos $\longrightarrow 50$ a 59 anos

\section{PORTO ALEGRE}

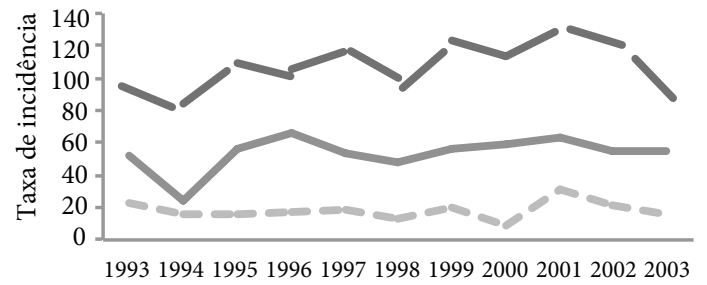

Ano

35 a 49 anos -25 a 34 anos -50 a 59 anos
2004, variando na duração do seguimento: Recife, 7 anos; São Paulo, 8 anos; Porto Alegre, 11 anos; Fortaleza, 13 anos. Na Tabela 1, encontra-se a síntese da análise estatística do estudo, conforme classificação na CID 10, estratificada por capital estudada. Complementarmente à referida tabela, na Figura 1 observa-se, por meio de análise gráfica, declínio na incidência do câncer do colo do útero invasor em Fortaleza e São Paulo, com respectiva significância estatística entre mulheres de 35 a 59 anos e mulheres de 50 a 59 anos. Conforme observado na Figura 2, a incidência de câncer de útero - porção não especificada - mostrou decréscimo entre mulheres de 25 a 59 anos na cidade de Porto Alegre, com significância estatística para aquelas entre 35 e 59 anos e limítrofe para as demais, e ainda comportamento similar entre as mulheres de Fortaleza (sem significância estatística, porém limítrofe para mulheres de 25 a 34 anos e 50 a 59 anos). Há tendência para crescimento da incidência do carcinoma in situ do colo do útero em todas as cidades e grupos etários estudados (Figura 3), com exceção de Recife, onde a representação gráfica dos dados mostrou grande irregularidade dos registros no período estudado, impossibilitando a análise proposta.

\section{RECIFE}

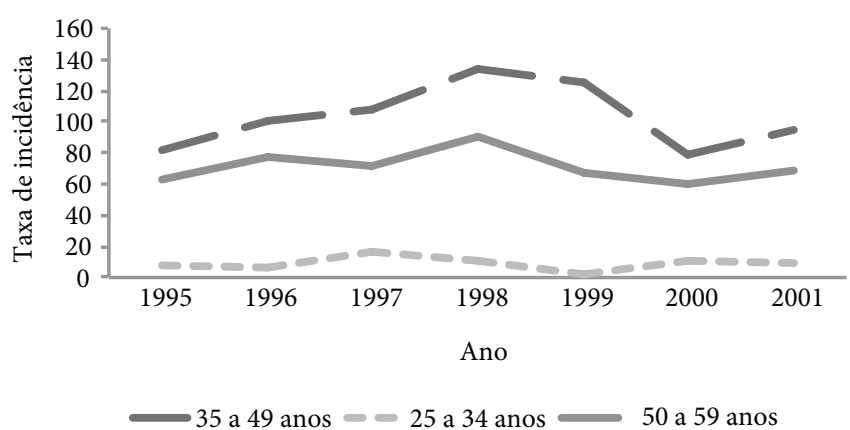

SÃO PAULO

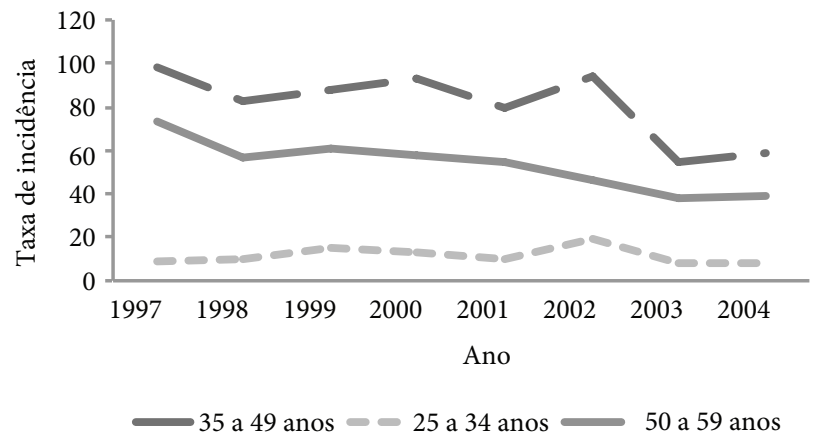

Figura 1. Série histórica das taxas de incidência de câncer de colo de útero invasor (C53) em capitais selecionadas - Brasil, 1990-2004 
FORTALEZA

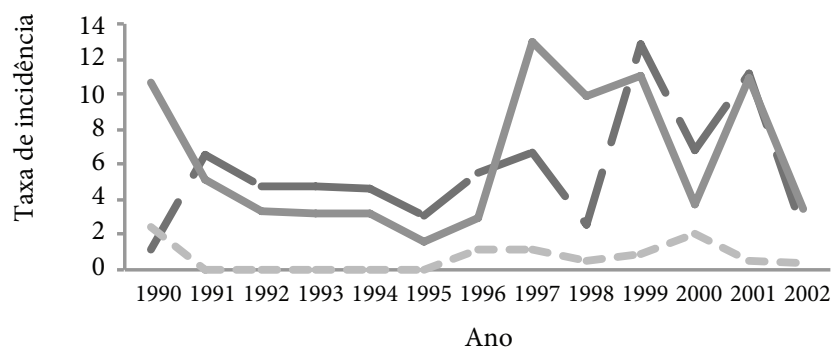

-25 a 34 anos -35 a 49 anos $\longrightarrow 50$ a 59 anos

PORTO ALEGRE

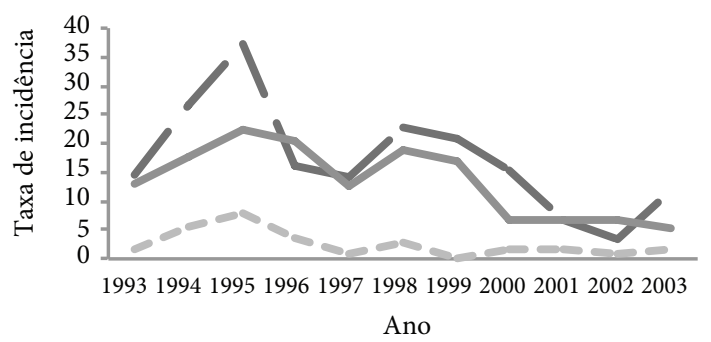

35 a 49 anos -25 a 34 anos -50 a 59 anos
RECIFE

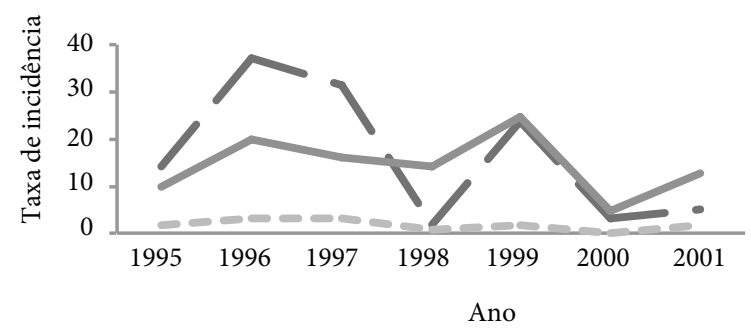

-35 a 49 anos -25 a 34 anos $\longrightarrow 50$ a 59 anos

SÃO PAULO

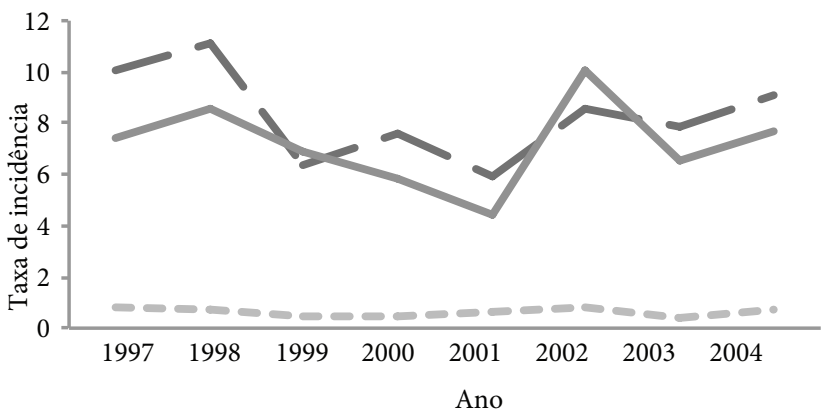

35 a 49 anos -25 a 34 anos 50 a 59 anos

Figura 2. Série histórica das taxas de incidência de câncer de colo do útero porção não especificada (C55) em capitais selecionadas - Brasil, 1990-2004

FORTALEZA

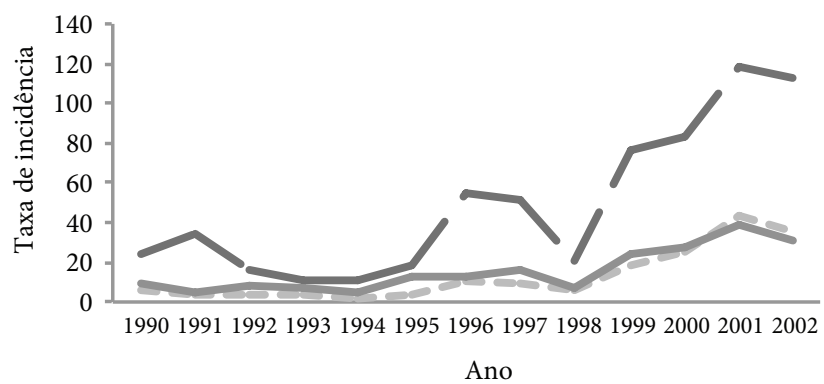

.25 a 34 anos -35 a 49 anos -50 a 59 anos

\section{PORTO ALEGRE}

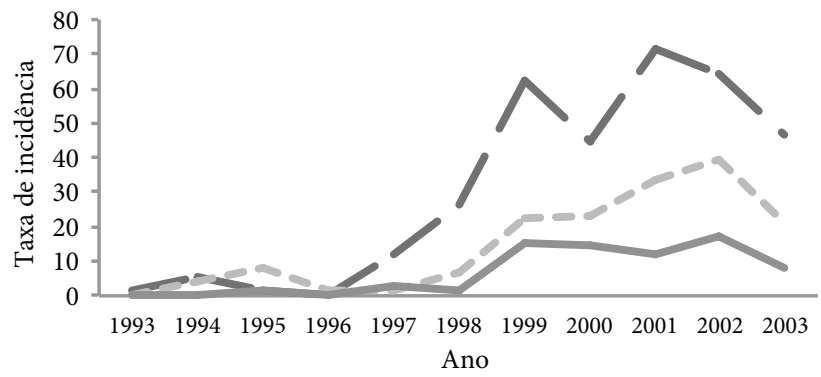

35 a 49 anos -25 a 34 anos -50 a 59 anos
RECIFE

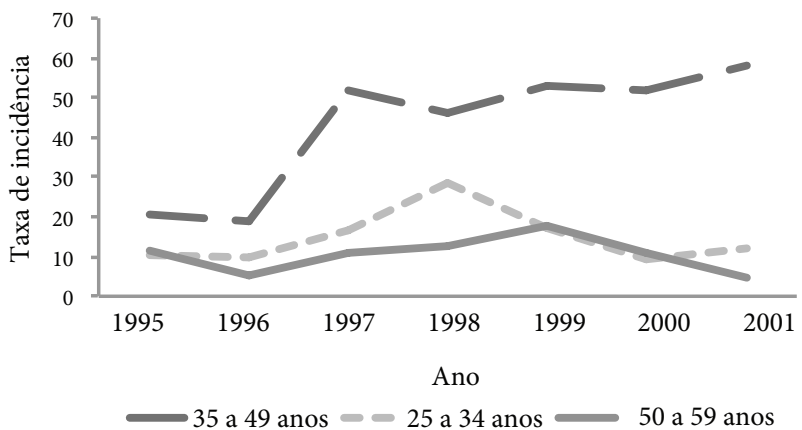

SÃO PAULO

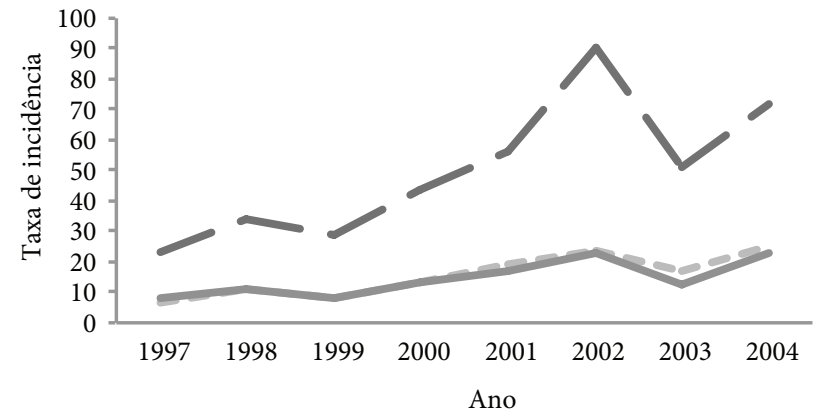

35 a 49 anos -25 a 34 anos 50 a 59 anos

Figura 3. Série histórica das taxas de incidência de câncer do colo do útero in situ (D06) em capitais selecionadas - Brasil, 1990-2004 
Tabela 1. Estatísticas da tendência segundo capital e CID-10 - Brasil, 2012

\begin{tabular}{|c|c|c|c|c|c|c|}
\hline CID 10 & Capital & Faixa etária & Modelo & Tendência & $\mathrm{R} 2$ & valor $\mathrm{p}$ \\
\hline \multirow{12}{*}{ C53 } & Fortaleza & $25-34$ & $f(x)=-5,594 x^{3}+0,752 x^{2}-0,030 x+22,547$ & Cúbica & 0,379 & 0,212 \\
\hline & & $35-49$ & $f(x)=-9,119 x+203,277$ & Linear & 0,730 & $<0,001$ \\
\hline & & $50-59$ & $f(x)=-4,532 x+137,231$ & Linear & 0,383 & 0,024 \\
\hline & Porto Alegre & $25-34$ & $f(x)=-8,611 x^{3}+1,566 x^{2}-0,080 x+29,453$ & Cúbica & 0,169 & 0,709 \\
\hline & & $35-49$ & $f(x)=-15,452 x^{3}+4,442 x^{2}-0,258 x+108,867$ & Cúbica & 0,541 & 0,122 \\
\hline & & $50-59$ & $\mathrm{f}(\mathrm{x})=\log _{6,242} \mathrm{x}+43,496$ & Logarítmica & 0,181 & 0,192 \\
\hline & Recife & $25-34$ & $f(x)=16,413 x^{3}+4,563 x^{2}+0,367 x-6,343$ & Cúbica & 0,240 & 0,815 \\
\hline & & $35-49$ & $f(x)=34,312 x^{2}-4,224 x+50,400$ & Quadrática & 0,575 & 0,181 \\
\hline & & $50-59$ & $f(x)=42,082 x^{3}-10,663 x^{2}+0,769 x+30,157$ & Cúbica & 0,502 & 0,498 \\
\hline & São Paulo & $25-34$ & $f(x)=4,339 x^{2}-0,492 x+4,761$ & Quadrática & 0,369 & 0,316 \\
\hline & & $35-49$ & $f(x)=-4,839 x+102,739$ & Linear & 0,531 & 0,040 \\
\hline & & $50-59$ & $f(x)=-4,493 x+73,393$ & Linear & 0,882 & 0,001 \\
\hline \multirow{12}{*}{ C55 } & Fortaleza & $25-34$ & $f(x)=-1,946 x^{3}+0,315 x^{2}-0,014 x+3,518$ & Cúbica & 0,635 & 0,023 \\
\hline & & $35-49$ & $\mathrm{f}(\mathrm{x})=\log _{1,853} \mathrm{x}+2,370$ & Logarítmica & 0,175 & 0,155 \\
\hline & & $50-59$ & $f(x)=-8,666 x^{3}+1,479 x^{2}-0,069 x+17,643$ & Cúbica & 0,540 & 0,062 \\
\hline & Porto Alegre & $25-34$ & $f(x)=-0,369 x+4,815$ & Linear & 0,280 & 0,094 \\
\hline & & $35-49$ & $f(x)=-0,128^{x}+31,446$ & Exponencial & 0,415 & 0,032 \\
\hline & & $50-59$ & $f(x)=-0,127^{x}+25,421$ & Exponencial & 0,622 & 0,004 \\
\hline & Recife & $25-34$ & $f(x)=-0,307 x+2,900$ & Linear & 0,323 & 0,183 \\
\hline & & $35-49$ & $f(x)=-3,700 x+31,457$ & Linear & 0,310 & 0,194 \\
\hline & & $50-59$ & $f(x)=6,145 x^{2}-0,826 x+6,500$ & Quadrática & 0,242 & 0,575 \\
\hline & São Paulo & $25-34$ & $f(x)=-0,077^{x}+0,727$ & Exponencial & 0,133 & 0,374 \\
\hline & & $35-49$ & $f(x)=-2,407 x^{2}+0,243 x+12,959$ & Quadrática & 0,546 & 0,139 \\
\hline & & $50-59$ & $f(x)=-1,010 x^{2}+0,112 x+8,864$ & Quadrática & 0,100 & 0,768 \\
\hline \multirow{12}{*}{ D06 } & Fortaleza & $25-34$ & $f(x)=0,494 x^{2}-4,094 x+10,220$ & Quadrática & 0,898 & $<0,001$ \\
\hline & & $35-49$ & $f(x)=1,224 x^{2}-9,074 x+34,972$ & Quadrática & 0,856 & $<0,001$ \\
\hline & & $50-59$ & $f(x)=0,298 x^{2}-1,792 x+9,336$ & Quadrática & 0,835 & $<0,001$ \\
\hline & Porto Alegre & $25-34$ & $f(x)=3,517 x-6,367$ & Linear & 0,716 & 0,001 \\
\hline & & $35-49$ & $f(x)=7,357 x+13,507$ & Linear & 0,757 & $<0,001$ \\
\hline & & $50-59$ & $f(x)=1,658 x-3,231$ & Linear & 0,619 & 0,004 \\
\hline & Recife & $25-34$ & $f(x)=9,869 x^{2}-1,210 x-0,486$ & Quadrática & 0,451 & 0,301 \\
\hline & & $35-49$ & $f(x)=\log _{21,282} x+16,867$ & Logarítmica & 0,796 & 0,007 \\
\hline & & $50-59$ & $f(x)=-17,088 x^{3}+5,849 x^{2}-0,542 x+22,529$ & Cúbica & 0,841 & 0,102 \\
\hline & São Paulo & $25-34$ & $\mathrm{f}(\mathrm{x})=2,558 \mathrm{x}+3,900$ & Linear & 0,814 & 0,002 \\
\hline & & $35-49$ & $f(x)=0,163^{x}+21,838$ & Exponencial & 0,747 & 0,006 \\
\hline & & $50-59$ & $f(x)=1,842 x+6,200$ & Linear & 0,584 & 0,027 \\
\hline
\end{tabular}

\section{DISCUSSÃO}

O estudo descreve o padrão de tendência temporal para a incidência do câncer do colo do útero com base nos RCBP. Embora os dados de Registros de Câncer de Base Populacional tenham como primeira função a avaliação de incidência, análises de tendência com base nestes devem ser vistas com cautela devido às dificuldades em se assegurar a mesma qualidade em cada ano. Mesmo assim, o declínio observado na incidência do câncer invasor e do câncer em porção não especificada em determinados grupos etários e o aumento da incidência do carcinoma in situ em todos os grupos estudados nos períodos descritos parece consistente.

De um modo geral, esse resultado vai ao encontro da tendência observada globalmente para o conjunto de países analisados pela Agência Internacional de Pesquisa do Câncer (IARC), ressaltando-se que o decréscimo se verifica em todos os países analisados, mas com magnitudes diferentes devido às altas taxas de incidência em países em desenvolvimento ${ }^{8}$.

A tendência para queda observada no câncer invasor e o crescimento do carcinoma in situ podem indicar resultados positivos das ações de rastreamento para o câncer do colo do útero nas cidades onde o fenômeno foi observado. O decréscimo de casos do carcinoma em porção não especificada aponta para melhora do diagnóstico com melhor descrição da localização da lesão.

Apesar das diferenças nos períodos de seguimento dos RCBP e na regularidade na obtenção dos dados, comprovou-se possível identificar um padrão de tendência temporal a partir 
dos dados analisados, em consonância com análise do RCBP de Goiânia, onde houve alcance dos objetivos propostos mesmo diante da complexidade do sistema de registros ${ }^{15}$.

As limitações presentes no estudo foram aquelas concernentes à utilização de fontes de dados secundários. Com relação aos RCBP, a passividade na obtenção dos dados pode aumentar o sub-registro de casos. Em determinado estudo com diferente delineamento, descreveu-se a necessidade de complementar dados com consulta ativa aos registros hospitalares para obtenção de dados de seguimento ${ }^{19}$.

É possível que a grande oscilação das taxas, especialmente para a cidade de Recife, possa ter relação com a irregularidade de obtenção de dados acarretada por aspectos vivenciados nos RCBP, como escassez de recursos humanos, materiais e administrativos ao longo do período estudado.

Os padrões de incidência para carcinoma in situ presentes nessa análise divergem, em alguns aspectos, da incidência apresentada pelo INCA9 . Ao contrário dos dados estimados para 2012, quando o grupo de mulheres entre 50 e 60 anos teria as maiores taxas de incidência, o grupo etário no qual se encontram as maiores taxas brutas de incidência foi o de 35 a 49 anos, com menores nas taxas a partir dos 50 anos. (dados não apresentados). Estudo realizado em município do interior do estado de São Paulo com alta cobertura de equipes da estratégia de saúde da família mostra que as mulheres com média de idade de 39 anos, seguidas das mulheres com 40 a 59 anos, são as que mais procuram as unidades para realização do exame preventivo, o que pode influenciar nas estimativas estratificadas por faixas etárias ${ }^{20}$. No Ceará, em um período de 5 anos na década de 1990, a maior proporção de casos de câncer de todos os tipos inseridos nos registros de câncer ocorreu entre mulheres de 40 a 69 anos, e não entre as mais jovens, com o câncer do colo do útero ocupando a segunda colocação ${ }^{21}$.

As taxas de incidência de câncer do colo do útero invasor de quatro RCBP brasileiros foram incluídas na última publicação da Agência Internacional de Pesquisa em Câncer (IARC), compreendendo estimativas anuais incluídas no período de 1998 a 2002. A inclusão dos RCBP selecionados pela IARC (Cuiabá, Goiânia, Brasília, São Paulo) se deu a partir de critérios de qualidade de informação e não conforme duração dos registros.
Apenas o RCBP de São Paulo foi selecionado pelo IARC e pelo presente estudo. A comparabilidade entre as estimativas deste estudo e as apresentadas pela IARC, no entanto, deve ser evitada, em razão das diferenças metodológicas utilizadas nas duas análises (taxa bruta de incidência versus taxa padronizada pela população mundial) e dos objetivos dos estudos, pois o IARC não analisou a tendência e sim estimativas pontuais com vistas à comparação das taxas entre mulheres de todo o mundo. Porém, mesmo com essas ressalvas, é interessante notar que a incidência nos dois estudos é elevada e se aproxima das taxas dos três países que apresentaram as mais altas incidências (Zimbábue, Uganda, Peru) ${ }^{22}$.

As informações produzidas pelos RCBP são fundamentais para definir o papel de fatores etiológicos e estabelecer prioridades na prevenção, no planejamento e no gerenciamento dos serviços de saúde voltados para o câncer. A melhoria da qualidade das informações dos RCBP no Brasil seria de grande importância na vigilância da incidência da doença.

\section{CONCLUSÃO}

A tendência para decréscimo observado no câncer invasor e o crescimento do carcinoma in situ entre grupos de mulheres estudadas por meio dos RCBP podem indicar resultados positivos das ações de rastreamento para o câncer do colo do útero nas cidades compreendidas neste estudo, exceto Recife. Os procedimentos adotados pelos RCBP devem ser reproduzidos em análises posteriores para aperfeiçoar o conhecimento sobre a epidemiologia do câncer do colo do útero. A irregularidade e a cobertura insuficiente dos dados registrados possivelmente podem interferir na qualidade das estimativas de incidência e na comparação do peso do câncer em diferentes regiões. No entanto, mesmo com essas limitações, as informações geradas por eles se mostram relevantes para o monitoramento do câncer. A contribuição dos registros foi mais significativa onde houve maior regularidade na captação e produção de informações. Melhorar as bases de dados é fundamental para potencializar a participação dos RCBP na vigilância do câncer no país e na definição de prioridades e estratégias de prevenção e controle.

\section{REFERÊNCIAS}

1. Brasil. Ministério da Saúde. Secretaria de Atenção à Saúde. Instituto Nacional de Câncer. Coordenação de Prevenção e Vigilância. Manual de rotinas e procedimentos para registros de câncer de base populacional. Rio de Janeiro: INCA, 2006.

2. Brasil. Ministério da Saúde. Registro de Cáncer en Brasil. Organización Panamericana de Salud. Boletín Epidemiológico. 1983;4(4):13-4.
3. Brasil. Ministério da Saúde. Instituto Nacional de Câncer. Câncer no Brasil. Dados dos Registros de Base Populacional. Histórico. 2003. [cited 2012 Mar 29]. Available from: http://www.inca.gov.br/regpop/2003/ index.asp?link=conteudo_view.asp\&ID $=13$

4. Brasil. Ministério da Saúde. Instituto Nacional de Câncer. Estatísticas do Câncer. Registros de Câncer de Base Populacional. Nota técnica. 
2009. [cited 2010 Feb 28]. Available from: http://wwwl.inca.gov.br/ cgi/sisbasepop.asp

5. Grupo de Registros e de Epidemiologia de Língua Latina. Estatutos 2006. [cited 2012 Feb 10]. Available from: http://www.grell-network.org/ home-1/grell_pt/quem-somos/estatuto

6. Brasil. Ministério da Saúde. Secretaria de Atenção à Saúde. Instituto Nacional de Câncer. Coordenação de Prevenção e Vigilância. A situação do câncer no Brasil. Rio de Janeiro: INCA, 2006.

7. Kligerman J. Registro hospitalar de câncer no Brasil. Revista Bras Cancerol. 2001;47(4):357-9.

8. Ferlay J, Shin HR, Bray F, Forman D, Mathers C, Parkin DM. GLOBOCAN 2008 v 1.2. Cancer Incidence and Mortality Worldwide: IARC CancerBase No. 10 [Internet]. Lyon, France: International Agency for Research on Cancer; 2010. [cited 2012 Jan 11]. Available from: http://globocan.iarc.fr

9. Brasil. Ministério da Saúde. Secretaria de Atenção à Saúde. Instituto Nacional de Câncer José Alencar Gomes da Silva. Coordenação Geral de Ações Estratégicas. Coordenação de Prevenção e Vigilância. Estimativa 2012: incidência de câncer no Brasil. Rio de Janeiro: Inca, 2011.

10. Azevedo e Silva G, Girianelli VR, Gamarra CJ, Bustamente-Teixeira MT. Evolução da mortalidade por câncer do colo do útero no Brasil, 19812006. Cad Saúde Pública. 2010;26(12):2399-407.

11. Muñoz N, Bosch FX, Sanjosé S, Herrero R, Castellsagué X, Shah K, Snijders PJF, Meijer CJLM. Epidemiologic classification of human papillomavirus types associated with cervical cancer. New Eng J Med. 2003; 348(6):518-27.

12. Walboomers JM, Jacobs MV, Manos MM, Bosch FX, Kummer JA, Shah KV, Snijders PJ, Peto J, Meijer CJ, Muñoz N. Human papillomavirus is a necessary cause of invasive cervical cancer worldwide. J Pathol. 1999;189(1):12-9.

13. Ayres ARG, Azevedo e Silva G. Prevalência de infecção do colo do útero pelo HPV no Brasil: revisão sistemática. Rev Saúde Pública. 2010;44(5):963-74.
14. Gamarra CJ, Valente JG, Azevedo e Silva G. Correção da magnitude da mortalidade por câncer do colo do útero no Brasil. Rev Saúde Pública. 2010;44(4):629-38.

15. Moura L, Curado MP, Simões EJ, Cezário AC, Urdaneta M. Avaliação do Registro de Câncer de Base Populacional do Município de Goiânia, Estado de Goiás, Brasil. Epidemiol Serv Saúde. 2006;15(4):7-17.

16. Reis RS, Santos MO, Thuler LCS. Incidência de tumores pediátricos no Brasil. Revista Bras Cancerol. 2007;53(1):5-15.

17. Organização Mundial da Saúde. Classificação Estatística Internacional de Doenças e Problemas Relacionados à Saúde: CID-10. Décima revisão. Tradução do Centro Colaborador da OMS para a Classificação de Doenças em Português. 10 ed. São Paulo: EDUSP, 2009.

18. Latorre MRDO, Cardoso MRA. Análise de séries temporais em epidemiologia: uma introdução sobre os aspectos epidemiológicos. Rev Bras Epidemiol. 2001;4(3):145-52.

19. Bustamante-Teixeira MT, Faerstein E, Mariotto A, Britto AV, Moreira Filho DC, Latorre MRDO. Sobrevida em pacientes com câncer gástrico em Campinas, São Paulo, Brasil. Cad Saúde Pública. 2006;22(8):1611-8.

20. Vale DBAP, Morais SS, Pimenta AL, Zeferino LC. Avaliação do rastreamento do câncer do colo do útero na Estratégia Saúde da Família no Município de Amparo, São Paulo, Brasil. Cad Saúde Pública. 2010;26(2):383-90.

21. Arregi MMU. Registro Hospitalar de Câncer: Cinco Anos de Experiência no Instituto do Câncer do Ceará, Brasil. Rev Bras Cancerol. 2000;46(4):377-87.

22. Curado MP, Edwards B, Shin HR, Storm H, Ferlay J, Heanue M, Boyle P. Cancer Incidence in Five Continents, Vol. IX. IARC Scientific Publications No. 160. Lyon: IARC, 2007.

Recebido em: 12/04/2012 Aprovado em: 28/07/2012 